ORIGINAL STUDY

\title{
Effectiveness of endoscopic posterior nasal neurectomy for the treatment of intractable rhinitis
}

\author{
Govindan Nair Arun1, Moideen P. Sanu', Mogarnad Mohan', Thampy S. Aparna², \\ Khizer Hussain Afroze M. ${ }^{3}$ \\ ${ }^{1}$ Department of Otorhinolaryngology, Sri Siddhartha Medical College, Tumakuru, Karnataka, India \\ ${ }^{2}$ Department of Dentistry, Padmavathy Medical Foundation, Kollam, Kerala, India \\ ${ }^{3}$ Department of Anatomy, Sri Siddhartha Medical College, Tumakuru, Karnataka, India
}

\section{ABSTRACT}

BACKGROUND. Chronic rhinitis is a clinical condition affecting more than $20 \%$ of the world population. The standard treatment strategy is medical. Surgical management can be considered in patients with intractable rhinitis. Various surgical techniques have been documented with varying success rates, but none of them is considered as a gold standard. Hence, we are studying the effectiveness of posterior nasal neurectomy (PNN) in patients who have intractable rhinitis, refractory to maximum medical therapy.

MATERIAL AND METHODS. A prospective study was conducted in the ENT Department, Padmavathy Medical Foundation, Kollam, Kerala, India, from January 2015 to February 2016. Adult patients, in the age group of 20 to 60 years, diagnosed with chronic rhinitis, presenting two or more symptoms of rhinitis, refractory to maximum medical therapy for a period of at least 3 or more years and whose quality of life was significantly affected were enrolled and PNN was done for them.

RESULTS. We observed a statistically significant improvement in subjective symptoms and patient quality of life at the end of 6 months post-operatively.

CONCLUSION. PNN is a safe and less invasive procedure, which can provide a significant relief in symptoms of intractable rhinitis. Fewer complications and better results make it superior over vidian neurectomy.

KEYWORDS: rhinitis, rhinorrhea, posterior nasal nerve, vidian neurectomy, RQLQ.

\section{INTRODUCTION}

Rhinitis is an inflammatory condition affecting the lining of the nose, characterized by nasal congestion, rhinorrhea, itching, sneezing and/or post-nasal discharge $^{1}$. More than $20 \%$ of the population is affected by chronic rhinitis ${ }^{2-4}$. Depending on whether an allergic etiology is implicated, non-infectious rhinitis can be subdivided into allergic and non-allergic. With 600 million of world's population affected, allergic rhinitis is the most prevalent atopic disorder. The incidence of allergic rhinitis is on steady increase ${ }^{5}$.

Various etiological factors have been postulated for chronic rhinitis - these include allergy, occupational exposures, hormonal changes, smoking, xylometazoline abuse, etc. The standard treatment strategy is medical (histamine antagonists, leukotriene receptor antagonists, intranasal corticosteroids, etc.) But many times, these therapies show limited effectiveness and cause substantial burden for treatment cost on the long term. In addition, the symptoms of intractable rhinitis can cause social embarrassment with a profound effect on patients' quality of life.

Surgical management can be considered in such patients with intractable rhinitis. Various surgical techniques have been documented including Vidian neurectomy $^{6}$, posterior nasal neurectomy, inferior turbinate reduction, with varying success rates $^{5-8}$. In view of this, we are studying the effectiveness of posterior nasal neurectomy (PNN) in patients who have intractable rhinitis, refractory to maximum medical therapy. The outcome is assessed with patient's level of satisfac- 
tion, with their pre and post-operative symptom scores using Rhino Conjunctivitis Quality of Life Questionnaire (RQLQ) adapted for the Indian population ${ }^{9-10}$.

The aim of this study was to evaluate the outcome of PNN in patients who have intractable chronic rhinitis by measuring subjective symptom improvement after surgery, and assess for possible complications.

\section{MATERIAL AND METHODS}

This prospective study was conducted in the ENT Department, Padmavathy Medical Foundation, Kollam, Kerala, from January 2015 to February 2016 (12 months).

\section{Patient selection}

Adult patients, in the age group of 20 to 60 years, diagnosed with chronic rhinitis, were enrolled for the study after obtaining the due consent. These include patients having two or more symptoms of rhinitis, refractory to maximum medical therapy for a period of at least 3 or more years and whose quality of life was significantly affected.

A diagnostic nasal endoscopy and a plain computed tomogram of the nose and paranasal sinuses were performed for all patients included in the study. Those patients with any anatomical variations (deviated nasal septum, hypertrophied turbinates, medialized uncinate process, polypoid nasal mucosa and sinonasal polyposis) were excluded from the study. Patients with history of previous sinonasal surgeries, and those with diabetes mellitus, hypertension, chronic heart disease and systemic causes of rhinitis (hormonal, drug induced) were also excluded from the study.

\section{Surgical procedure}

Posterior nasal neurectomy was performed in those patients who were eligible for the study after applying the inclusion criteria. All selected patients were instructed to stop all antirhinitis medications 1 month prior to surgery.

The procedure can be done either under general anaesthesia or local anaesthesia. We prefer hypotensive general anaesthesia (Mean Arterial Pressure - 55 to $65 \mathrm{mmHg}$ ) in reverse Trendelenburg position. Local anaesthesia is not preferred due to the possibility of bleeding from the sphenopalatine artery. After induction of general anaesthesia and oral intubation, the nasal cavity was packed with 1:1000 adrenaline patties for local vasoconstriction. All surgical procedures were performed with a $0^{0}$ or $30^{\circ}, 4 \mathrm{~mm}$ Storz nasal endoscope (Karl Storz, Tuttlingen, Germany).

The surgical procedure was similar to that of transnasal endoscopic sphenopalatine artery ligation ${ }^{10}$. An incision carried deep down to the bone is made on the lateral nasal wall, at the level of the posterior fontanelle, with a number 15 surgical blade or flag knife used in micro ear surgery. The mucosal flap was elevated posteriorly till the posterior end of the middle meatus, until the fibroneurovascular sleeve including the sphenopalatine artery and the posterior nasal nerve arising from the sphenopalatine foramen were identified. The crista ethmoidalis is a landmark for the sphenopalatine foramen ${ }^{11}$.

Identification of the posterior nasal nerve: The proximal portion or the main trunk of the nerve lies anterior to the sphenopalatine artery at the sphenopalatine foramen level. The nerve is carefully delineated and cauterized using a bipolar cautery and it is resected with micro scissors. The transected nerve fiber can be confirmed by the histopathological examination. The nerve is resected at its main trunk, in order to avoid missing of its peripheral branches.

The mucoperiosteal flap was repositioned, sufficient coagulations done to achieve hemostasis and nasal packing was performed with surgical or Merocel® sponge. Patients were given oral antibiotics, analgesics and antihistamines for 5 days postoperatively.

Complications: Hemorrhage if injury to sphenopalatine vessels, infections and nasal adhesions which are generally less common in experienced hands.

\section{Follow-up}

All patients were reviewed postoperatively after the first week, second week, first month, third month and sixth month. Patient feedbacks were collected during the first-month, third-month and sixth-month visits. No anti-rhinitis medications were given during the follow-up periods.

\section{Subjective evaluation and quality of life}

The patients were evaluated 2 weeks before surgery and during the first, second and sixth month postoperatively.

Subjective evaluation was performed with Okuda's system, a numerical score based system where each symptom (average daily number of sneezes, frequency of rhinorrhea, degree of nasal obstruction and degree of total severity) is scored on a scale of 0 to 4 (0-none, 1-mild, 2-moderate, 3-severe, 4 -very severe $)^{12}$.

The quality of life was assessed using a regionally and culturally validated (Indian) version of Rhino Conjunctivitis Quality of Life Questionnaire (RQLQ). This Questionnaire has 28 questions in 7 domains - activity limitation, sleep problems, nose symptoms, eye symptoms, non-nose/eye symptoms, practical problems and emotional factors. Three patient-specific questions in the activity domain allow them to select three activities in which they are most limited due to rhinitis. Each item is rated on a seven-point scale ranging from 0 (not 
impaired at all) to six (severely impaired). The average RQLQ score is calculated by taking the mean of all 28 responses. A change of at least 0.5 in RQLQ score is considered to be of clinical significance $\mathrm{e}^{8,13}$.

\section{Statistical analysis}

The data obtained was put into a master chart and it was assessed using the IBM SPSS V16 software. The variables were presented as mean \pm SD. Post-operative improvement in symptom scores were evaluated with Wilcoxon signed-rank test. A p-value $<0.05$ was considered to be statistically significant.

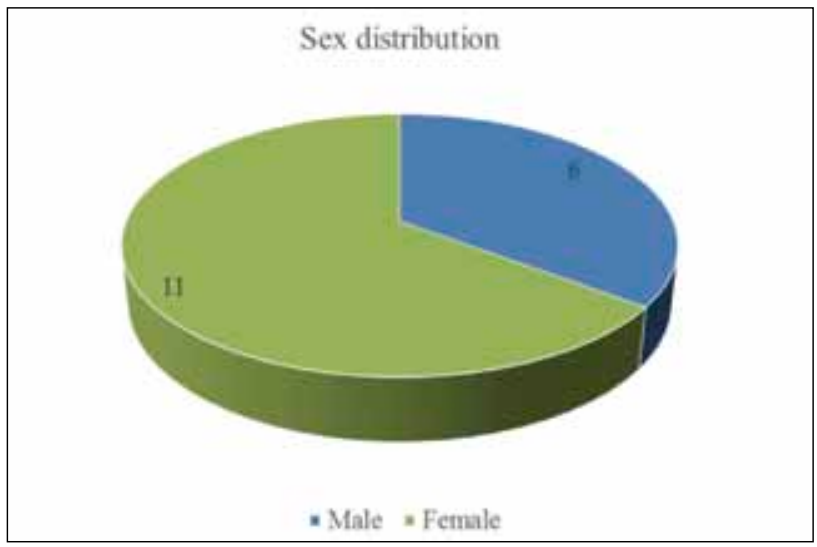

Figure 1 Sex distribution in the study population.

\section{RESULTS}

During our study period from January 2015 to February 2016, 19 patients were enrolled for the study. 2 patients were lost for the 6-month follow-up; hence we excluded them from the study group. There were 6 male patients $(35.29 \%)$ and 11 female patients $(64.70 \%)$ (Figure 1$)$, age range of 27 to $52(36.24 \pm$ 7.93) (Figure 2).

Subjective nasal symptoms of all 17 patients improved over a period of 6 months (Table 1, Figure 3). The mean symptom scores for sneezing, rhinorrhea,

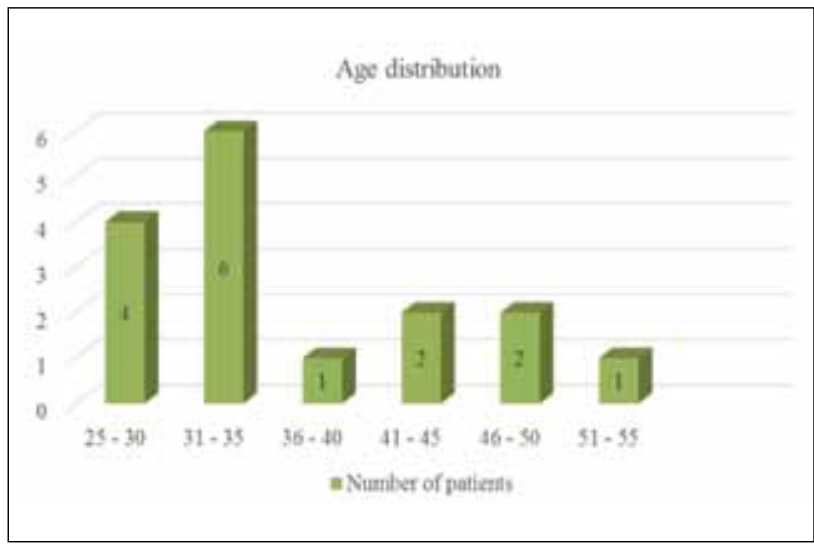

Figure 2 Age distribution in the study population.

Table 1

Subjective nasal symptom scores evaluation with Okuda's score before and after surgery

\begin{tabular}{ccccc}
\hline Time period & Sneezing & Rhinorrhea & Nasal obstruction & Total severity \\
\hline Pre-0p & $3.235 \pm 0.562$ & $2.647 \pm 0.320$ & $2.882 \pm 0.781$ & $3.647 \pm 0.492$ \\
\hline $1^{\text {st }}$ month & $1.117 \pm 0.857$ & $0.647 \pm 0.492$ & $1.352 \pm 0.606$ & $1.882 \pm 0.332$ \\
\hline $3^{\text {rd }}$ month & $0.882 \pm 0.781$ & $0.058 \pm 0.242$ & $0.4117 \pm 0.795$ & $0.647 \pm 0.606$ \\
\hline $6^{\text {th }}$ month & $0.941 \pm 0.428$ & $0.058 \pm 0.042$ & $0.294 \pm 0.587$ & $0.4117 \pm 0.507$ \\
\hline Pre- $0 p$ vs $6^{\text {th }}$ month post-operatively & $0.0011^{* *}$ & $0.0038^{* *}$ & $0.0171^{*}$ & $0.0026^{* *}$ \\
\hline${ }^{*}$ p value $\leq 0.05,{ }^{* *}$ p value $\leq 0.01$ & & &
\end{tabular}

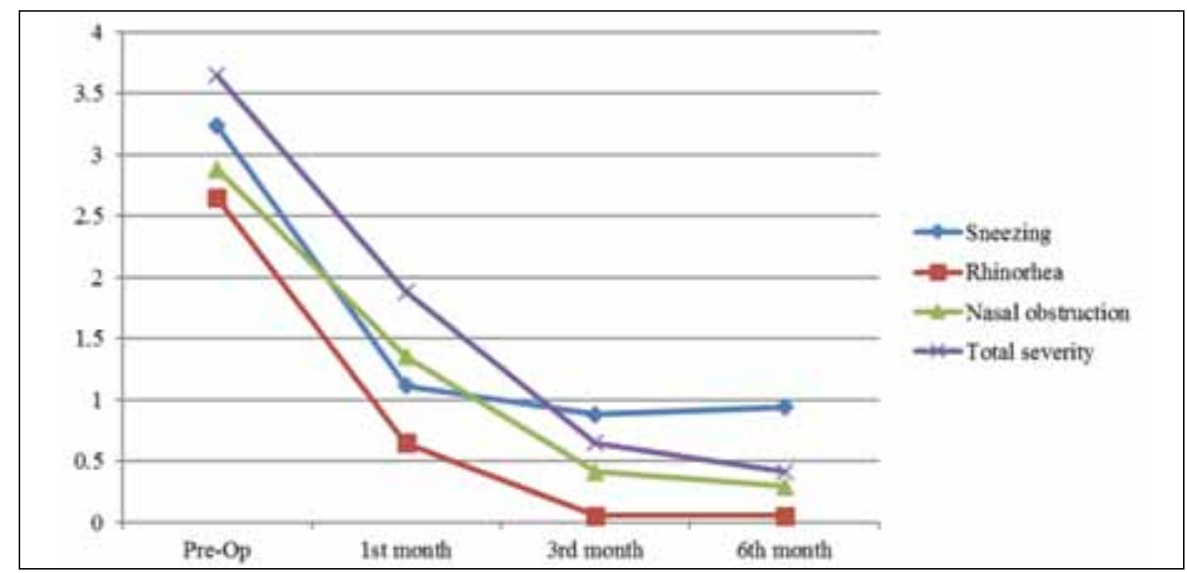

Figure 3 Mean time-dependent subjective symptom score. 
nasal obstruction and total severity were all decreased from pre-operative levels after the third and sixth month postoperatively. The $p$-values for these were statistically significant $(<0.01)$.

We observed a significant improvement in patients' quality of life at the end of 6 months post-operatively (Table 2, Figure 4). The changes in all 7 domains of RQLQ were statistically significant.

No major complications including bleeding from the sphenopalatine artery or its branches, severe postoperative pain, dry eyes, dry mouth, numbness of cheek or palate related to the procedure were noted.
Crusting or atrophy of the turbinates was not seen in any of the patients. Adhesions were noted in 4 cases postoperatively, which were released during the follow-up periods.

\section{DISCUSSIONS}

It was Golding-Wood who first described vidian neurectomy (transantral approach) for vasomotor rhinitis (VMR) in 1960. The vidian nerve (or nerve of the pterygoid canal) provides the main post ganglionic

Table 2

Changes in RQLQ scores after treatment

\begin{tabular}{cllr}
\hline Parameters & Pre-0p & $\mathbf{6}^{\text {th }}$ Month & $\begin{array}{c}\text { Intragroup difference before } \\
\text { and after treatment }\end{array}$ \\
\hline Activities & $3.51 \pm 1.24$ & $1.82 \pm 1.01$ & $0.0061^{* *}$ \\
\hline Sleep & $2.91 \pm 1.13$ & $1.60 \pm 1.32$ & $0.0069^{* *}$ \\
\hline Non-hay fever symptoms & $2.74 \pm 1.07$ & $1.79 \pm 0.98$ & $0.0030^{* *}$ \\
\hline Practical problems & $3.78 \pm 1.29$ & $2.14 \pm 1.17$ & $0.0018^{* *}$ \\
\hline Nasal symptoms & $3.94 \pm 1.31$ & $1.69 \pm 0.53$ & $0.0378^{*}$ \\
\hline Eye symptoms & $2.84 \pm 1.27$ & $1.02 \pm 0.61$ & $0.0411^{*}$ \\
\hline Emotions & $3.42 \pm 1.53$ & $2.11 \pm 1.24$ & $0.0159^{*}$ \\
\hline Overall & $3.97 \pm 1.27$ & $1.32 \pm 0.41$ & $0.0334^{*}$ \\
\hline${ }^{*}$ value $\leq 0.05{ }^{* *} p$ value $\leq 0.01$ & & &
\end{tabular}

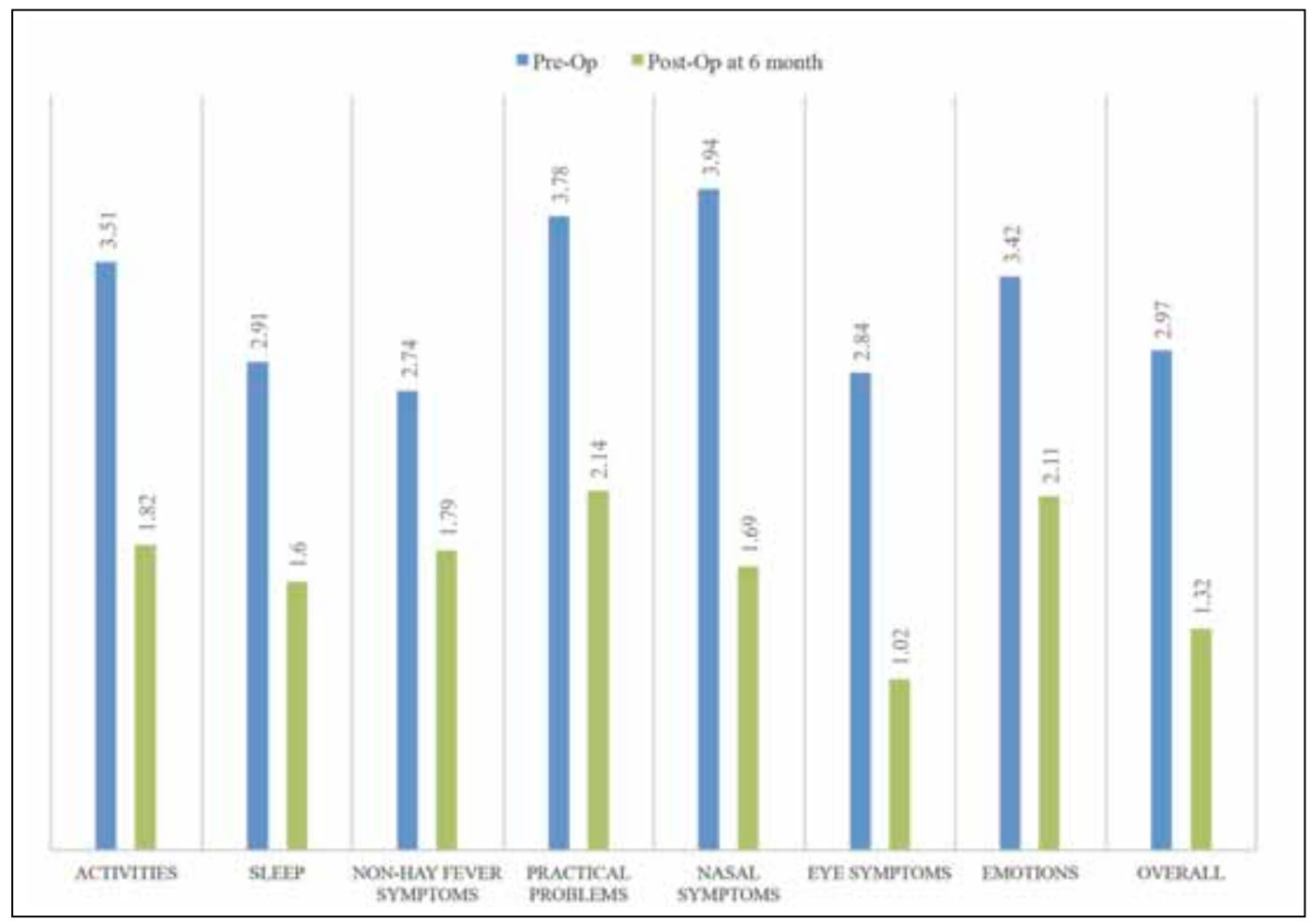

Figure 4 Mean Pre-Op and Post-Op scores with RQLQ 
parasympathetic supply to the nasal mucosa, the palate and to the lacrimal gland. Stimulation of the vidian nerve can cause secretory and vasodilator effects in humans as well as animals. Resection of the vidian nerve can reduce the hyperactivity of the nasal reflex, which alleviates sneezing and nasal hypersecretion ${ }^{14}$.

The last two decades have shown tremendous advancement in the field of endoscopic sinus surgeries. Robinson and Wormold described techniques for endoscopic vidian neurectomy and reported that rhinorrhea and nasal obstruction were significantly improved after endoscopic vidian neurectomy. They also described the histological changes following vidian neurectomy - significant reduction of stromal edema and eosinophilic infiltration, reduction of mast cell and histamine and reduction of mucosal acinar gland cells. This is likely to have been caused by the interruption of cholinergic innervations to the nasal mucosa following transaction of the efferent pathway of the parasympathetic reflex ${ }^{15}$.

Later, the procedure of vidian neurectomy was almost abandoned due to technical difficulties in approaching the pterygopalatine region, significant associated complications including severe bleeding from the sphenopalatine artery and its branches, dry eyes due to decreased lacrimation, ophthalmoplegia and even blindness ${ }^{15-17}$.

Microanatomic studies performed by Ruskell showed that a secretory motor fiber exits the pterygopalatine foramen (PPF) in multiple rami, each coursing to a different target (e.g. lacrimal gland and nasal mucosa). The ramus originating from the pterygopalatine ganglion (PPG) is found to specifically innervate the nasal mucosa and it has been called the posterior nasal nerve. Selective resection of this posterior nasal nerve abolishes parasympathetic supply to the nasal cavity and provides the same benefits of vidian neurectomy, without having any of its complications $^{18,19}$.

Ikeda et al. ${ }^{20}$ observed that posterior nasal neurectomy causes suppression of the secretagogue motor and inhibition of the neurogenic inflammation induced by the parasympathetic and sensory denervation. Ogawa et al. ${ }^{21}$ found out that PNN in allergic rhinitis patients significantly reduce levels of IL-5, eotaxin protein in nasal secretions. They also observed reduction of infiltrated immuno-component cells in the subepithelial mucous layer, which are major sources of cytokine release.

Mori et al. ${ }^{22}$, Kobayashi et al. ${ }^{23}$ also reported similar patient benefits following posterior nasal neurectomy. They concluded that selective resection of peripheral branches of the posterior nerve could reduce allergic symptoms. Kawamura et al. ${ }^{24}$, in their study of PNN with harmonic scalpel among 20 patients, observed subjective improvement in nasal obstruction, sneezing and nasal discharge in $100 \%, 90 \%$ and $75 \%$ patients respectively. They reported no surgical complications.

In our study, we found that the mean score for each nasal symptom of all patients was statistically decreased from pre-operative levels at the six-month follow-upwithout any major complications.

We selected RQLQ for evaluation of quality of life because it is a standardized and validated method. The use of RQLQ in measuring quality of life in epidemiological surveys, clinical trials and patient monitoring are well documented ${ }^{11,13}$.

Limitations of our study: This was a short-term study on a limited number of cases. We could not do an objective evaluation of patients' symptoms.

Studies with longer follow-up periods and a large number of patients are suggested to validate our results.

\section{CONCLUSIONS}

Endoscopic resection of the posterior nasal nerve is a safe and less invasive procedure, which can provide a significant relief in symptoms of intractable rhinitis, particularly rhinorrhea and nasal obstruction. Fewer complications and better results make it superior over vidian neurectomy.

Acknowledgments: We thank Elizabeth F. Juniper, MCSP, MSc, Professor Emeritus, Department of Clinical Epidemiology and Biostatistics, McMaster University, Canada \& Mrs. Penny Freeman, QOL Technologies limited, UK, for providing us with regionally validated versions of Rhino Conjunctivitis Quality of Life Questionnaire.

Conflict of interest: We declare no conflict of interest. No sponsorship was made for this study.

Contribution of authors: All authors have equally contributed to this work.

Ethical approval: All procedures performed in studies involving human participants were in accordance with the ethical standards of the institutional and/or national research committee and with the $1964 \mathrm{Hel}-$ sinki declaration and its later amendments or comparable ethical standards

\section{REFERENCES}

1. International Consensus Report on the Diagnosis and Management of Rhinitis. International Rhinitis Management Working Group. Allergy, 1994;49(19 Suppl):1-34.

2. Carney A.S., Jones N.S. - Idiopathic rhinitis: idiopathic or not? Clin Otolaryngol Allied Sci., 1996;21 (3):198-202.

3. Schünemann H.J., et al. - Allergic Rhinitis and its Impact on Asthma (ARIA) 2010 Revision.

4. Bousquet J., Schünemann H.J., Samolinski B., Demoly P., Baena-Cagnani 
C.E., Bachert C., Bonini S., Boulet L.P., Bousquet P.J., Brozek J.L., Canonica G.W., et al. - Allergic Rhinitis and its Impact on Asthma (ARIA): achievements in 10 years and future needs. J Allergy Clin Immunol., 2012;130(5):1049-1062. doi: 10.1016/j.jaci.2012.07.053. Epub 2012 Oct 4.

5. Chhabra N., Houser S.M. - Surgical options for the allergic rhinitis patient. Current Opinion in Otolaryngology \& Head \& Neck Surgery, 2012;20(3):199-204.

6. Golding-Wood P.H. - Observations on petrosal and vidian neurectomy in chronic vasomotor rhinitis. J Laryngol. Otol., 1961;75:232-247.

7. Halderman A., Sindwani R. - Surgical management of vasomotor rhinitis: a systematic review. Am J Rhinol Allergy, 2015;29(2):128-134. doi: 10.2500/ajra.2015.29.4141.

8. Juniper E.F., Guyatt G.H., Griffith L.E., Ferrie P.J. - Interpretation of rhinoconjunctivitis quality of life questionnaire data. J Allergy Clin Immunol., 1996;98(4):843-845.

9. Park K.H., Cho J.S., Lee K.H., Shin S.Y., Moon J.H., Cha C.I. Rhinoconjunctivitis quality of life questionnaire (RQLQ) as an evaluator of perennial allergic rhinitis patients-the first report. Korean J Otolaryngology-Head Neck Surg., 2002;45(3):254-262.

10. Sharp H.R., Rowe-Jones J.M., Biring G.S., Mackay I.S. - Endoscopic ligation or diathermy of the sphenopalatine artery in persistent epistaxis. J Laryngol Otol., 1997;111(11):1047-1050.

11. Bolger W.E., Borgie R.C., Melder P. - The role of the crista ethmoidalis in endoscopic sphenopalatine artery ligation. Am J Rhinol., 1999;13(2):8186.

12. Okuda M., Ishikawa T., Saito Y., Shimizu T., Baba S. - A clinical evaluation of $\mathrm{N}-5$ 'with perennial-type allergic rhinitis-a test by the multi-clinic, intergroup, double-blind comparative method. Ann Allergy, 1984;53(2):178-185.

13. Juniper E.F., Guyatt G.H., Willan A., Griffith L.E. - Determining a minimal important change in a disease-specific Quality of Life Questionnaire. J Clin Epidemiol., 1994;47(1):81-87.

14. Konno A. - Historical, pathophysiological, and therapeutic aspects of vidian neurectomy. Current Allergy and Asthma Reports, 2010;10(2):105-112.
15. sl Shazly M.A. - Endoscopic surgery of the vidian nerve. Preliminary report. Ann Otol Rhinol Laryngol., 1991;100(7):536-539.

16. el-Guindy A. - Endoscopic transseptal vidian neurectomy. Arch Otolaryngol Head Neck Surg., 1994;120(12):1347-1351.

17. Lee J.C., Lin Y.S. - Endoscopic vidian neurectomy: update on techniques and evidence. Curr Opin Otolaryngol Head Neck Surg., 2012;20(1):6672. doi: 10.1097/MOO.0b013e32834e13d8.

18. Ruskell G.L. - Orbital passage of pterygopalatine ganglion efferents to paranasal sinuses and nasal mucosa in man. Cells Tissues Organs, 2004;175(4):223-228.

19. Ruskell G.L. - Distribution of pterygopalatine ganglion efferents to the lacrimal gland in man. Experimental Eye Research, 2004;78(3):329-335.

20. Ikeda K., Yokoi H., Saito T., Kawano K., Yao T., Furukawa M. - Effect of resection of the posterior nasal nerve on functional and morphological changes in the inferior turbinate mucosa. Acta Oto-laryngol., 2008;128(12):1337-1341. doi: 10.1080/00016480801935525.

21. Ogawa T., Takeno S., Ishino T., Hirakawa K. - Submucous turbinectomy combined with posterior nasal neurectomy in the management of severe allergic rhinitis: clinical outcomes and local cytokine changes. Auris Nasus Larynx, 2007;34(3):319-326. Epub 2007 Apr 11.

22. Mori S., Fujieda S., Igarashi M., Fan G.K., Saito H. - Submucous turbinectomy decreases not only nasal stiffness but also sneezing and rhinorrhea in patients with perennial allergic rhinitis. Clin Exp Allergy, 1999;29:15421548.

23. Kobayashi T., Hyodo M., Nakamura K., Komobuchi H., Honda N. Resection of peripheral branches of the posterior nasal nerve compared to conventional posterior neurectomy in severe allergic rhinitis. Auris Nasus Larynx, 2012;39(6):593-596. doi: 10.1016/j.anl.2011.11.006. Epub $2012 \mathrm{Feb} 15$.

24. Kawamura S., Asako M., Momotani A., Kedai H., Kubo N., Yamashita T. - Submucosal turbinectomy with posterior-superior nasal neurectomy for patients with allergic rhinitis. Pract Oto Rhino Laryngol., 2000;93(5):367372. 